\title{
Existence and uniqueness of measure solutions for a system of continuity equations with non-local flow
}

\author{
Gianluca Crippa and Magali Lécureux-Mercier
}

\begin{abstract}
In this paper, we prove existence and uniqueness of measure solutions for the Cauchy problem associated to the (vectorial) continuity equation with a non-local flow. We also give a stability result with respect to various parameters.
\end{abstract}

Mathematics Subject Classification. 35L65.

Keywords. System of conservation laws, Continuity equations, Wasserstein distance, Nonlocal flow, Pedestrian traffic.

\section{Introduction}

In this paper, we consider the system of nonlocal continuity equations

$$
\begin{cases}\partial_{t} \rho^{i}+\operatorname{div}\left(\rho^{i} V^{i}\left(t, x, \eta^{i} * \rho\right)\right)=0, & t \in \mathbb{R}^{+}, x \in \mathbb{R}^{d} \\ \rho^{i}(0)=\bar{\rho}^{i}, & i \in\{1, \ldots, k\}\end{cases}
$$

where the unknown $\rho=\left(\rho^{1}, \ldots, \rho^{k}\right)$ is a vector of measures, $\eta^{i}=\left(\eta^{i, 1}, \ldots, \eta^{i, k}\right)$ for each $i \in\{1, \ldots, k\}$ is a vector of convolution kernels and we set $\eta^{i} * \rho=$ $\left(\eta^{i, 1} * \rho^{1}, \ldots, \eta^{i, k} * \rho^{k}\right)$. For any time $t \geqslant 0$, if $\mu_{t} \in \mathcal{M}^{+}\left(\mathbb{R}^{d}\right)$ is a bounded measure on $\mathbb{R}^{d}$ and $\eta_{t}$ is a bounded function on $\mathbb{R}^{d}$, then the convolution is taken with respect to space variables only and is defined as usually as $\left(\mu_{t} * \eta_{t}\right)(x)=$ $\int_{\mathbb{R}^{d}} \eta_{t}(x-y) \mathrm{d} \mu_{t}(y)$.

Equations with this structure can describe sedimentation models, supply-chains, or pedestrian traffic (see later in this introduction more precise descriptions of these models, and references to the literature). For physical reasons, in the following we are looking for positive solutions, since we intend to describe the time evolution of a density (for instance, of pedestrians or of some physical quantity). Moreover, we are interested in allowing concentrations (for instance, in points or along hypersurfaces) of the density. Hence, we 
will consider (vector valued) solutions such that for any time each component belongs to the space $\mathcal{M}^{+}\left(\mathbb{R}^{d}\right)$ of positive measures with finite total mass.

In this paper we prove existence and uniqueness for the system (1.1), together with stability estimates with respect to various parameters, and further properties of the solutions. We consider the following structural hypotheses:

(V) The vector field $V(t, x, r): \mathbb{R}^{+} \times \mathbb{R}^{d} \times \mathbb{R}^{k} \rightarrow \mathbf{M}_{d, k}$ is uniformly bounded and it is Lipschitz in $(x, r) \in \mathbb{R}^{d} \times \mathbb{R}^{k}$ uniformly in time:

$$
V \in \mathbf{L}^{\infty}\left(\mathbb{R}^{+} \times \mathbb{R}^{d} \times \mathbb{R}^{k}\right) \cap \mathbf{L}^{\infty}\left(\mathbb{R}^{+}, \operatorname{Lip}\left(\mathbb{R}^{d} \times \mathbb{R}^{k}, \mathbf{M}_{d, k}\right)\right) .
$$

( $\boldsymbol{\eta}$ ) The convolution kernel $\eta(t, x): \mathbb{R}^{+} \times \mathbb{R}^{d} \rightarrow \mathbf{M}_{k}$ is uniformly bounded and it is Lipschitz in $x \in \mathbb{R}^{d}$ uniformly in time:

$$
\eta \in \mathbf{L}^{\infty}\left(\mathbb{R}^{+} \times \mathbb{R}^{d}\right) \cap \mathbf{L}^{\infty}\left(\mathbb{R}^{+}, \operatorname{Lip}\left(\mathbb{R}^{d}, \mathbf{M}_{k}\right)\right) .
$$

Our main result is the following theorem.

Theorem 1.1. Let $\bar{\rho} \in \mathcal{M}^{+}\left(\mathbb{R}^{d}\right)^{k}$. Let us assume that $V$ satisfies $(\mathbf{V})$ and $\eta$ satisfies $(\boldsymbol{\eta})$. Then there exists a unique solution $\rho \in \mathbf{L}^{\infty}\left(\mathbb{R}^{+}, \mathcal{M}^{+}\left(\mathbb{R}^{d}\right)^{k}\right)$ to (1.1) with initial condition $\bar{\rho}$.

We refer to Sect. 2 for precise notations and definitions, in particular for the notion of solution.

Remark 1.2. If $V$ satisfies $(\mathbf{V})$ and $\eta$ satisfies $(\boldsymbol{\eta})$, in addition to the wellposedness provided by Theorem 1.1 we have the following further properties:

- If $\bar{\rho} \in \mathbf{L}^{1}\left(\mathbb{R}^{d},\left(\mathbb{R}^{+}\right)^{k}\right)$ then $\rho \in \mathscr{C}^{0}\left(\mathbb{R}^{+}, \mathbf{L}^{1}\left(\mathbb{R}^{d},\left(\mathbb{R}^{+}\right)^{k}\right)\right)$, up to redefinition on a negligible set of times; for all time $t \geqslant 0$ and for all $i \in\{1, \ldots, k\}$ we have $\left\|\rho^{i}(t)\right\|_{\mathbf{L}^{1}}=\left\|\bar{\rho}^{i}\right\|_{\mathbf{L}^{1}}$.

- If $\bar{\rho} \in\left(\mathbf{L}^{1} \cap \mathbf{L}^{\infty}\right)\left(\mathbb{R}^{d},\left(\mathbb{R}^{+}\right)^{k}\right)$ then $\rho \in \mathbf{L}_{\text {loc }}^{\infty}\left(\mathbb{R}^{+}, \mathbf{L}^{\infty}\left(\mathbb{R}^{d},\left(\mathbb{R}^{+}\right)^{k}\right)\right)$ and for all time $t \geqslant 0$, we have $\|\rho(t)\|_{\mathbf{L}^{\infty}} \leqslant\|\bar{\rho}\|_{\mathbf{L}^{\infty}} e^{C t}$, with $C$ a constant dependent on $\operatorname{Lip}_{x}(V), \operatorname{Lip}_{r}(V), \operatorname{Lip}_{x}(\eta)$ and $\|\bar{\rho}\|_{\mathcal{M}}$.

- Let $\bar{\rho}, \bar{\sigma} \in \mathcal{M}^{+}\left(\mathbb{R}^{d}\right)^{k}$ such that for any $i,\left\|\bar{\rho}^{i}\right\|_{\mathcal{M}}=\left\|\bar{\sigma}^{i}\right\|_{\mathcal{M}}$. Let $\rho$ and $\sigma$ be the solutions of (1.1) associated to the initial conditions $\bar{\rho}$ and $\bar{\sigma}$. Then we have the estimate:

$$
\mathscr{W}_{1}\left(\rho_{t}, \sigma_{t}\right) \leqslant e^{K t} \mathscr{W}_{1}(\bar{\rho}, \bar{\sigma}),
$$

where $K=\operatorname{Lip}_{x}(V)+\operatorname{Lip}_{r}(V) \operatorname{Lip}_{x}(\eta)\|\bar{\rho}\|_{\mathcal{M}}+\operatorname{Lip}_{r}(V) \operatorname{Lip}_{x}(\eta)\|\bar{\rho}\|_{\mathcal{M}}$ and $\mathscr{W}_{1}\left(\rho_{t}, \sigma_{t}\right)$ is the Wasserstein distance of order one between $\rho_{t}$ and $\sigma_{t}$.

As we pointed out before, the use of measure solutions allows the treatment of concentrations in the evolving density. However, the first two observations in this remark clarify that concentrations cannot be produced by the dynamics of our problem, when starting with diffuse initial data. Measure solutions are useful to describe the evolution of concentrated initial data, which may persist to be concentrated under the time evolution.

The above properties are described in Corollary 2.9 and in Proposition 4.2. The Wasserstein distance of order one is defined in Sect. 3. 
Remark 1.3. In Theorem 1.1 as well as in the other results of this papers, it is in fact sufficient to require that $V^{i}(t, x, r)$ is $\mathbf{L}^{\infty}$ in $t, x$ and $\mathbf{L}_{l o c}^{\infty}$ in $r$. Indeed, $\rho * \eta^{i}$ is uniformly bounded by $\|\bar{\rho}\|_{\mathcal{M}}\|\eta\|_{\mathbf{L}^{\infty}}=M$. Consequently, denoting $B_{M}$ the closed ball of center 0 and radius $M$ in $\mathbb{R}^{k}$, it is sufficient to require $V^{i} \in \mathbf{L}^{\infty}\left(\mathbb{R}^{+} \times \mathbb{R}^{d} \times B_{M}\right)$.

Note also that, restricting the definition of $V$ and $\eta$ to the time interval $\left[0, T_{0}\right]$, we obtain a solution defined on the same time interval. Consequently, we can as well just require $V$ and $\eta$ to be $\mathbf{L}_{l o c}^{\infty}$ in time instead of $\mathbf{L}^{\infty}$.

\subsection{A model of pedestrian traffic}

System (1.1) stands for a variety of models. Let us present first a macroscopic model of pedestrian traffic. In a macroscopic pedestrian crowd model, $\rho$ is the density of the crowd at time $t$ and position $x$ and $V$ is a vector field giving the speed of the pedestrians. According to the choice of $V$, various behaviors can be observed. Several authors already studied pedestrian traffic in two space dimensions. Some of these models are local in $\rho$ (see $[4,11,17,18,20,21])$; other models use not only the local density $\rho(t, x)$ but the entire distribution of $\rho$, typically they depend on the convolution product $\rho(t) * \eta$ (see $[6-9,14,23]$ ) which represents the spatial average of the density. Within the framework of (1.1), we can study the models presented in $[6,8,9]$. In [8], the authors considered for $V$ the expression

$$
V=v(\rho * \eta) \vec{v}(x),
$$

where $v$ is a scalar function giving the speed of the pedestrians; $\eta$ is a convolution kernel averaging the density, and $\vec{v}(x)$ is a bounded vector field giving the direction the pedestrian located in $x$ will follow. This model is more adapted to the case of panic in which pedestrians will not deviate from their trajectory and will adapt their velocity to the averaged density. Indeed, even if the density is maximal on a given trajectory, if the averaged density is not maximal, the pedestrians will push, trying all the same to reach their goal. This behavior can be associated with rush phenomena in which people can even die due to overcompression (e.g. on Jamarat Bridge in Saudi Arabia, see [16]).

The authors of [8] study the scalar case in the framework of Kružkov entropy solutions. They obtaine existence and uniqueness of weak entropy solutions under the hypotheses $v \in \mathbf{W}^{2, \infty}\left(\mathbb{R}^{+}, \mathbb{R}^{+}\right), \vec{v} \in\left(\mathbf{W}^{2, \infty} \cap \mathbf{W}^{2,1}\right)\left(\mathbb{R}^{d}, \mathbb{R}^{d}\right)$, and $\eta \in\left(\mathbf{W}^{2, \infty} \cap \mathbf{L}^{1}\right)\left(\mathbb{R}^{d}, \mathbb{R}\right)$. This result was slightly improved in [9] where, under the same set of hypotheses on $v, \vec{v}$ and $\eta$, the authors consider a system instead of a scalar equation and obtain global in time existence and uniqueness of entropy solutions. In the present paper, we recover and improve these results, assuming lighter hypotheses. Indeed, although we consider weak measure solutions, these in fact are unique and consequently coincide with the entropy solutions of $[8,9]$ when the initial condition is in $\mathbf{L}^{1}$.

A related model was studied in $[12,23]$ where the authors, instead of an isotropic convolution kernel, consider a nonlocal functional taking into account the direction in which the pedestrians are looking. Results in a somehow similar spirit to the present paper have been obtained in [22], where the authors 
restrict to the case of absolutely continuous measures, and also present various related numerical schemes.

\subsection{Coupling diffuse populations and isolated agents}

Another model of crowd dynamics that we recover consists in the coupling of a group of density $\rho(t, x)$ with an isolated agent located in $p(t)$. This can modelize for example the interaction between groups of preys of densities $\rho$ and an isolated predator located in $p$. Such a model was introduced in [6] where the authors obtained existence and uniqueness of weak entropy solutions under very strong hypotheses.

We recover here partially the results concerning the coupling PDE/ODE of [6]. Indeed, the measure framework allows us also to introduce particles/individuals through Dirac measures.

For instance, let us assume that $k=k_{0}+k_{1}$ such that $\rho^{1}, \ldots, \rho^{k_{0}}$ are in fact functions belonging to $\mathbf{L}^{\infty}\left(\mathbb{R}^{+}, \mathbf{L}^{1}\left(\mathbb{R}^{d}, \mathbb{R}^{+}\right)\right)$and that $\delta_{p^{1}}, \ldots, \delta_{p^{k_{1}}}$ are Dirac measures located in $p^{1}(t), \ldots, p^{k_{1}}(t) \in \mathbb{R}^{d}$. Let us denote $\rho=$ $\left(\rho^{1}, \ldots, \rho^{k_{0}}\right) \in \mathbb{R}^{k_{0}}$ and $p=\left(p^{1}, \ldots, p^{k_{1}}\right) \in \mathbf{M}_{d, k_{1}}$. We also denote with $V^{i}$ (resp., $\eta^{i}$ ) the vector fields (resp., kernels) associated to $\rho$, and with $U^{i}$ (resp., $\lambda^{i}$ ) the vector fields (resp., kernels) associated to $p$. Note that $\delta_{p^{j}} * \lambda^{i, j}(x)=\lambda^{i, j}\left(x-p^{j}\right)$. By definition of weak measure solution (see Definition 2.2), if $p_{i} \in \mathscr{C}^{1}\left([0, T], \mathbb{R}^{d}\right)$, the Dirac measures are satisfying, for any $i \in\left\{1, \ldots, k_{1}\right\}$

$\dot{p}_{i}(t)=U^{i}\left[t, p_{i}(t), \rho_{t} * \eta_{t}^{i}\left(p_{i}(t)\right), \lambda^{i, 1}\left(p_{i}(t)-p_{1}(t)\right), \ldots, \lambda^{i, k_{1}}\left(p_{i}(t)-p_{k_{1}}(t)\right)\right]$,

which can be rewritten

$$
\dot{p}^{i}(t)=\Phi^{i}\left(t, p(t), \rho_{t} * \eta_{t}^{i}\left(p^{i}(t)\right)\right) .
$$

Consequently, in this case, system (1.1) becomes

$$
\begin{cases}\partial_{t} \rho^{i}+\operatorname{div}\left(\rho^{i} V^{i}\left(t, x, \eta_{t}^{i} * \rho_{t},\left(\lambda^{i, j}\left(x-p^{j}(t)\right)\right)_{j=1}^{k_{1}}\right)\right)=0, & i \in\left\{1, \ldots, k_{0}\right\}, \\ \dot{p}^{j}(t)=\Phi^{j}\left(t, p(t), \rho_{t} * \eta_{t}^{j}\left(p^{j}(t)\right)\right), & j \in\left\{1, \ldots, k_{1}\right\},\end{cases}
$$

a coupling of ODEs with conservation laws.

\subsection{Further models}

The system (1.1) comprises also a model of particles sedimentation, introduced in [24] and studied in [26], where the author proves existence and uniqueness of weak solutions with initial condition in $\mathbf{L}^{\infty}$. A related nonlocal model is the supply-chain model $[2,3]$, in which the integral $\int_{0}^{1} \rho(t, x) \mathrm{d} x$ replaces the convolution product. This last model was studied for example in [10], imposing boundary conditions in $x=0$ and $x=1$.

In the context of Hamiltonian systems, very general existence results, together with approximation schemes, have been obtained in [1]: however, uniqueness seems to be out of reach in such low regularity context. Models of aggregation are studied in [5], using gradient flows techniques and allowing singular kernels. 


\subsection{Strategy of the proof and plan of the paper}

The proof of Theorem 1.1 is divided into two main steps. First, we prove some a priori properties of the solutions (see Sect. 2): mainly, we prove that the weak measure solutions of (1.1) coincide with the Lagrangian solutions of this system. Important consequences are the conservation of the regularity of the initial condition and the strong continuity in time in the case the solution is a function, as stated in Remark 1.2.

Second, we prove the existence and uniqueness of Lagrangian solutions thanks to a fixed point argument (see Sect. 5). Indeed, introducing the set of probability measures endowed with the Wasserstein distance of order one, we are able to prove a stability estimate with respect to the nonlocal term (see Sect. 4). The technique used there is quite similar to the one of Loeper [19], who studied the Vlasov-Poisson equation and the Euler equation in vorticity formulation.

This article is organized as follows: in Sect. 2 we define the two different notions of solution and prove that they coincide. In Sect. 3 we give some useful tools on optimal tranport. In Sect. 4 we prove an important lemma giving a stability estimate. Finally, in Sect. 5, we give the proof of Theorem 1.1.

\section{Notion of solutions}

\subsection{General notations}

Let $d \in \mathbb{N}$ be the space dimension and $k \in \mathbb{N}$ be the size of the system. In the following, $\mathbf{M}_{d, k}$ is the set of matrices of size $d \times k$ with real values and $\mathbf{M}_{k}$ is the set of matrices of size $k \times k$ with real values. In all computations, we will consider the 1-norm (i.e., the sum of the absolute values of the entries) on both vectors and matrices. When considering other norms, a constant depending on $d$ and/or $k$ appears in the estimates.

We denote by $\mathcal{M}\left(\mathbb{R}^{d}\right)$ (resp., $\mathcal{M}^{+}\left(\mathbb{R}^{d}\right)$ ) the set of finite mass (resp., finite mass and positive) measures on $\mathbb{R}^{d}$ and by $\mathcal{P}\left(\mathbb{R}^{d}\right)$ the set of probability measures on $\mathbb{R}^{d}$, that is the set of bounded positive measures with total mass 1. If $\rho=\left(\rho^{1}, \ldots, \rho^{k}\right) \in \mathcal{M}\left(\mathbb{R}^{d}\right)^{k}$, we define the total mass of $\rho$ as $\|\rho\|_{\mathcal{M}}=$ $\left\|\rho^{1}\right\|_{\mathcal{M}}+\cdots+\left\|\rho^{k}\right\|_{\mathcal{M}}$.

In the following the Lipschitz norms with respect to $x$ or $r$ are taken uniformly with respect to the other variables. That is to say, for example:

$$
\operatorname{Lip}_{x}(V)=\sup _{t \in \mathbb{R}^{+}, r \in \mathbb{R}^{k}}\left\{\operatorname{Lip}_{x}(V(t, \cdot, r))\right\}
$$

The space $\mathbf{L}^{\infty}\left([0, T], \mathcal{M}^{+}\left(\mathbb{R}^{d}\right)\right)$ consists of the parametrized measures $\mu=$ $\left(\mu_{t}\right)_{t \in[0, T]}$ such that, for any $\phi \in \mathscr{C}_{c}^{0}\left(\mathbb{R}^{d}, \mathbb{R}\right)$, the application $t \mapsto \int_{\mathbb{R}^{d}} \phi \mathrm{d} \mu_{t}(x)$ is measurable and such that ess $\sup _{t \in[0, T]}\left\|\mu_{t}\right\|_{\mathcal{M}}<\infty$.

\subsection{Weak measure solutions}

We say that $\rho \in \mathbf{L}^{\infty}\left([0, T], \mathcal{M}^{+}\left(\mathbb{R}^{d}\right)^{k}\right)$ is a weak measure solution of (1.1) with initial condition $\bar{\rho} \in \mathcal{M}^{+}\left(\mathbb{R}^{d}\right)^{k}$ if, for any $i \in\{1, \ldots, k\}$ and for any test-function $\phi \in \mathscr{C}_{c}^{\infty}(]-\infty, T\left[\times \mathbb{R}^{d}, \mathbb{R}\right)$ we have 


$$
\int_{0}^{T} \int_{\mathbb{R}^{d}}\left[\partial_{t} \phi+V^{i}\left(t, x, \rho * \eta^{i}\right) \cdot \nabla \phi\right] \mathrm{d} \rho_{t}^{i}(x) \mathrm{d} t+\int_{\mathbb{R}^{d}} \phi(0, x) \mathrm{d} \bar{\rho}^{i}(x)=0 .
$$

Remark 2.3. A priori for weak measure solutions of the continuity equation $\partial_{t} \rho+\operatorname{div}(\rho b)=0$, with a given vector field $b$, we have only continuity in time for the weak topology (see [13]), that is to say, for all $i \in\{1, \ldots, k\}$, for all $\phi \in \mathscr{C}_{c}^{0}\left(\mathbb{R}^{d}, \mathbb{R}\right)$, the application $t \mapsto \int_{\mathbb{R}^{d}} \phi(x) \mathrm{d} \rho_{t}^{i}(x)$ is continuous, up to redefinition of $\rho_{t}$ on a negligible set of times.

In the case of the system (1.1), we have a gain of regularity in time when the initial condition is a function in $\mathbf{L}^{1}\left(\mathbb{R}^{d},\left(\mathbb{R}^{+}\right)^{k}\right)$ (see Corollary 2.9).

\subsection{Push-forward and change of variable}

When $\mu$ is a measure on $\Omega$ and $T: \Omega \rightarrow \Omega^{\prime}$ a measurable map, we denote $T_{\sharp} \mu$ the push-forward of $\mu$, that is the measure on $\Omega^{\prime}$ such that, for every $\phi \in \mathscr{C}_{c}^{0}\left(\Omega^{\prime}, \mathbb{R}\right)$,

$$
\int_{\Omega^{\prime}} \phi(y) \mathrm{d} T_{\sharp} \mu(y)=\int_{\Omega} \phi(T(x)) \mathrm{d} \mu(x) .
$$

If we assume that $\mu$ and $\nu=T_{\sharp} \mu$ are absolutely continuous with respect to the Lebesgue measure, so that there exist $f, g \in \mathbf{L}^{1}$ such that $\mathrm{d} \mu(x)=f(x) \mathrm{d} x$ and $\mathrm{d} \nu(y)=g(y) \mathrm{d} y$, and that $T$ is a Lip-diffeomorphism, then we have the change of variable formula

$$
f(x)=g(T(x))|\operatorname{det}(\nabla T(x))| .
$$

Besides, we denote by $\mathbb{P}_{x}: \mathbb{R}^{d} \times \mathbb{R}^{d} \rightarrow \mathbb{R}^{d}$ the projection on the first coordinate; that is, for any $(u, v) \in \mathbb{R}^{d} \times \mathbb{R}^{d}, \mathbb{P}_{x}(u, v)=u$. In a similar way, $\mathbb{P}_{y}: \mathbb{R}^{d} \times \mathbb{R}^{d} \rightarrow \mathbb{R}^{d}$ is the projection on the second coordinate; that is, for any $(u, v) \in \mathbb{R}^{d} \times \mathbb{R}^{d}, \mathbb{P}_{y}(u, v)=v$.

\subsection{Lagrangian solutions}

We say that $\rho \in \mathbf{L}^{\infty}\left([0, T], \mathcal{M}^{+}\left(\mathbb{R}^{d}\right)^{k}\right)$ is a Lagrangian solution of (1.1) with initial condition $\bar{\rho} \in \mathcal{M}^{+}\left(\mathbb{R}^{d}\right)^{k}$ if, for any $i \in\{1, \ldots, k\}$, there exists an ODE flow $X^{i}:[0, T] \times \mathbb{R}^{d} \rightarrow \mathbb{R}^{d}$, that is a solution of

$$
\left\{\begin{array}{l}
\frac{\mathrm{d} X^{i}}{\mathrm{~d} t}(t, x)=V^{i}\left(t, X^{i}(t, x), \rho_{t} * \eta_{t}^{i}\left(X^{i}(t, x)\right)\right), \\
X^{i}(0, x)=x,
\end{array}\right.
$$

such that $\rho_{t}^{i}=X_{t \sharp}^{i} \bar{\rho}^{i}$ where $X_{t}^{i}: \mathbb{R}^{d} \rightarrow \mathbb{R}^{d}$ is the map defined as $X_{t}^{i}(x)=$ $X^{i}(t, x)$ for any $(t, x) \in[0, T] \times \mathbb{R}^{d}$.

Remark 2.6. Assume $V$ satisfies $(\mathbf{V})$ and $\eta$ satisfies $(\boldsymbol{\eta})$. Then, for any $\rho \in$ $\mathbf{L}^{\infty}\left([0, T], \mathcal{M}^{+}\left(\mathbb{R}^{d}\right)^{k}\right)$, the vector fields $b=V\left(t, x, \rho_{t} * \eta_{t}\right)$ are Lipschitz in $x$ and

$$
\operatorname{Lip}_{x}(b) \leqslant \operatorname{Lip}_{x}(V)+\operatorname{Lip}_{r}(V) \operatorname{Lip}_{x}(\eta)\left\|\rho_{t}\right\|_{\mathcal{M}} .
$$

Consequently, if $\left\|\rho_{t}\right\|_{\mathcal{M}}$ is uniformly bounded, the ODE flow $X^{i}$ above is always well-defined, for a fixed $\rho$. 
If $\bar{\rho} \in \mathbf{L}^{1}\left(\mathbb{R}^{d}, \mathbb{R}^{+}\right)$, then the push-forward formula (2.1) becomes, for a.e. $(t, x) \in \mathbb{R}^{+} \times \mathbb{R}^{d}$,

$\rho^{i}\left(t, X^{i}(t, x)\right)=\bar{\rho}^{i}(x) \exp \left(-\int_{0}^{t} \operatorname{div} V^{i}\left(\tau, X^{i}(\tau, x), \rho_{\tau} * \eta_{\tau}\left(X^{i}(\tau, x)\right)\right) \mathrm{d} \tau\right)$.

We now show that the two notions of solution in fact coincide.

Theorem 2.7. If $\rho$ is a Lagrangian solution of (1.1), then $\rho$ is also a weak measure solution of (1.1). Conversely, if $\rho$ is a weak measure solution of (1.1), then $\rho$ is also a Lagrangian solution of (1.1).

Proof. Step 1. Let $\rho$ be a Lagrangian solution of (1.1). Let us denote $b^{i}=$ $V^{i}\left(t, x, \rho * \eta^{i}\right)$ and let $X^{i}$ be the ODE flow associated to $b^{i}$. Then, for any $\phi \in \mathscr{C}_{c}^{\infty}(]-\infty, T\left[\times \mathbb{R}^{d}, \mathbb{R}\right)$, we have

$$
\begin{aligned}
\int_{0}^{T} & \int_{\mathbb{R}^{d}}\left(\partial_{t} \phi(t, x)+b^{i}(t, x) \cdot \nabla \phi(t, x)\right) \mathrm{d} \rho_{t}(x) \mathrm{d} t \\
& =\int_{0}^{T} \int_{\mathbb{R}^{d}}\left(\partial_{t} \phi\left(t, X_{t}^{i}(x)\right)+b^{i}\left(t, X_{t}^{i}(x)\right) \cdot \nabla \phi\left(t, X_{t}^{i}(x)\right)\right) \mathrm{d} \bar{\rho}(x) \mathrm{d} t \\
& =\int_{0}^{T} \int_{\mathbb{R}^{d}} \frac{\mathrm{d}}{\mathrm{d} t}\left(\phi\left(t, X_{t}^{i}(x)\right)\right) \mathrm{d} \bar{\rho}(x) \mathrm{d} t \\
& =\int_{\mathbb{R}^{d}} \phi\left(T, X^{i}(T, x)\right) \mathrm{d} \bar{\rho}(x)-\int_{\mathbb{R}^{d}} \phi(0, x) \mathrm{d} \bar{\rho}(x)=-\int_{\mathbb{R}^{d}} \phi(0, x) \mathrm{d} \bar{\rho}(x),
\end{aligned}
$$

which proves that $\rho$ is also a weak measure solution.

Step 2. Let $\rho$ be a weak measure solution of (1.1). For any $i \in\{1, \ldots, k\}$, let us denote $b^{i}(t, x)=V^{i}\left(t, x, \rho * \eta^{i}\right)$. Let $\sigma$ be the Lagrangian solution of the equation

$$
\partial_{t} \sigma^{i}+\operatorname{div}\left(\sigma^{i} b^{i}\right)=0, \quad \sigma^{i}(0, \cdot)=\bar{\rho}^{i}
$$

which exists and is unique since $b^{i}$ is Lipschitz as noted in Remark 2.6. Then, arguing similarly as in point $1, \sigma$ is also a weak measure solution to (2.3). Denoting $u^{i}=\rho^{i}-\sigma^{i}$, we obtain that $u^{i}$ is a weak measure solution of the equation $\partial_{t} u^{i}+\operatorname{div}\left(u^{i} b^{i}\right)=0$ with initial condition $u^{i}(0, \cdot)=0$. Consequently, for any $\phi \in \mathscr{C}_{c}^{\infty}(]-\infty, T\left[\times \mathbb{R}^{d}, \mathbb{R}\right)$,

$$
\int_{0}^{T} \int_{\mathbb{R}^{d}}\left(\partial_{t} \phi+b^{i}(t, x) \cdot \nabla \phi\right) \mathrm{d} u_{t} \mathrm{~d} t=0 .
$$

Let $\psi \in \mathscr{C}_{c}^{0}(]-\infty, T\left[\times \mathbb{R}^{d}, \mathbb{R}\right)$. Since $b^{i} \in \mathbf{L}^{\infty}\left([0, T] \times \mathbb{R}^{d}, \mathbb{R}^{d}\right)$ is Lipschitz in $x$, by computation along the characteristics, we can find $\phi \in \mathscr{C}_{c}^{1}$ (] $\infty, T\left[\times \mathbb{R}^{d}, \mathbb{R}\right)$ so that $\psi=\partial_{t} \phi+b^{i}(t, x) \cdot \nabla \phi$. Hence, for any $\psi \in \mathscr{C}_{c}^{0}(]-$ $\infty, T\left[\times \mathbb{R}^{d}, \mathbb{R}\right)$, we have $\int_{0}^{T} \int_{\mathbb{R}^{d}} \psi \mathrm{d} u_{t} \mathrm{~d} t=0$, which implies $u \equiv 0$ a.e., and so $\rho \equiv \sigma$ a.e. Consequently, we have also $b^{i}(t, x)=V^{i}\left(t, x, \sigma * \eta^{i}\right)$, and $\sigma=\rho$ is finally a Lagrangian solution of (1.1). 
Definition 2.8. As a consequence of the previous theorem, in the following we simply call solution of (1.1) a weak measure solution or a Lagrangian solution of (1.1), since these two notions in fact coincide.

It is now possible to prove some of the properties given in Remark 1.2.

Corollary 2.9. Assume that $V$ satisfies (V) and $\eta$ satisfies $(\boldsymbol{\eta})$. Let $\rho \in$ $\mathbf{L}^{\infty}\left([0, T], \mathcal{M}^{+}\left(\mathbb{R}^{d}\right)^{k}\right)$ be a solution to (1.1) with initial condition $\bar{\rho} \in$ $\mathcal{M}^{+}\left(\mathbb{R}^{d}\right)^{k}$.

- If $\bar{\rho} \in \mathbf{L}^{1}\left(\mathbb{R}^{d},\left(\mathbb{R}^{+}\right)^{k}\right)$. Then we have $\rho \in \mathscr{C}^{0}\left([0, T], \mathbf{L}^{1}\left(\mathbb{R}^{d},\left(\mathbb{R}^{+}\right)^{k}\right)\right)$ and for all time $t \in[0, T]$, all $i \in\{1, \ldots, k\},\left\|\rho^{i}(t)\right\|_{\mathbf{L}^{1}}=\left\|\bar{\rho}^{i}\right\|_{\mathbf{L}^{1}}$.

- If furthermore $\bar{\rho} \in\left(\mathbf{L}^{1} \cap \mathbf{L}^{\infty}\right)\left(\mathbb{R}^{d},\left(\mathbb{R}^{+}\right)^{k}\right)$, then for all $t \in[0, T]$ we have $\rho(t) \in \mathbf{L}^{\infty}\left(\mathbb{R}^{d},\left(\mathbb{R}^{+}\right)^{k}\right)$ and we have the estimate

$$
\|\rho(t)\|_{\mathbf{L}^{\infty}} \leqslant\|\bar{\rho}\|_{\mathbf{L}^{\infty}} e^{C t}
$$

where $C$ depends on $\|\bar{\rho}\|_{\mathcal{M}}, V$ and $\eta$.

Proof. Let $\rho$ be a solution of (1.1) with initial condition $\bar{\rho} \in \mathbf{L}^{1}\left(\mathbb{R}^{d},\left(\mathbb{R}^{+}\right)^{k}\right)$. According to Definition 2.8, $\rho$ is a Lagrangian solution associated to a flow $X$ and we have immediately that $\|\bar{\rho}\|_{\mathbf{L}^{1}}=\|\rho(t)\|_{\mathbf{L}^{1}}$.

Besides, as $b^{i}(t, x)=V^{i}\left(t, x, \rho * \eta^{i}\right) \in \mathbf{L}^{\infty}\left([0, T] \times \mathbb{R}^{d}, \mathbb{R}^{d}\right)$ is bounded in $t$ and Lipschitz in $x$, then $X_{t}^{i} \in \operatorname{Lip}\left(\mathbb{R}^{d}, \mathbb{R}^{d}\right)$ and we can use the change of variable formula (2.2). If $\bar{\rho} \in \mathbf{L}^{\infty}\left(\mathbb{R}^{d}, \mathbb{R}^{k}\right)$, with

$$
C=\operatorname{Lip}_{x}(V)+\operatorname{Lip}_{r}(V) \operatorname{Lip}_{x}(\eta)\|\bar{\rho}\|_{\mathcal{M}},
$$

we obtain the desired $\mathbf{L}^{\infty}$ bound and $\rho(t) \in \mathbf{L}^{\infty}$ for all $t \in[0, T]$.

The continuity in time can be proved directly by estimating $\left\|\rho_{t}-\rho_{s}\right\|_{\mathbf{L}^{1}}$ using Egorov Theorem. This computation is straightforward although a bit long so we prefer to omit the details. Alternatively, note that the continuity in time is also ensured by the results of DiPerna and Lions [15, Sect. 2.II] and the notion of renormalized solutions.

\section{Some tools from optimal mass transportation}

Let us remind the definition of the Wasserstein distance of order 1.

Definition 3.1. Let $\mu, \nu$ be two Borel probability measures on $\mathbb{R}^{d}$. We denote $\Xi(\mu, \nu)$ the set of plans, that is the set of probability measures $\gamma \in \mathcal{M}^{+}\left(\mathbb{R}^{d} \times\right.$ $\mathbb{R}^{d}$ ) such that $\mathbb{P}_{x \sharp} \gamma=\mu$ and $\mathbb{P}_{y_{\sharp}} \gamma=\nu$. We define the Wasserstein distance of order one between $\mu$ and $\nu$ by

$$
W_{1}(\mu, \nu)=\inf _{\gamma \in \Xi(\mu, \nu)} \int_{\mathbb{R}^{d} \times \mathbb{R}^{d}}|x-y| \mathrm{d} \gamma(x, y) .
$$

Let $\rho=\left(\rho^{1}, \ldots, \rho^{k}\right), \sigma=\left(\sigma^{1}, \ldots, \sigma^{k}\right)$ be two vectors such that $\rho^{1}, \ldots, \rho^{k}$ and $\sigma^{1}, \ldots, \sigma^{k}$ are Borel probability measures on $\mathbb{R}^{d}$. We define the Wasserstein distance of order one between $\rho$ and $\sigma$, denoted $\mathscr{W}_{1}(\rho, \sigma)$, as

$$
\mathscr{W}_{1}(\rho, \sigma)=\sum_{i=1}^{k} W_{1}\left(\rho^{i}, \sigma^{i}\right) .
$$


Remark 3.2. By [25, Theorem 1.3] for any $\mu, \nu \in \mathcal{P}\left(\mathbb{R}^{d}\right)$, there exist a plan $\gamma_{0} \in \Xi(\mu, \nu)$ realizing the minimum in the Wasserstein distance, that is

$$
W_{1}(\mu, \nu)=\int_{\mathbb{R}^{d}}|x-y| \mathrm{d} \gamma_{0}(x, y) .
$$

Remark 3.3. Let $\bar{\rho} \in \mathcal{M}^{+}\left(\mathbb{R}^{d}\right)^{k}$ be a probability measure and let $X, Y: \mathbb{R}^{d} \rightarrow$ $\mathbb{R}^{d}$ be mappings such that $f=X_{\sharp} \mathrm{d} \bar{\rho}$ and $g=Y_{\sharp} \mathrm{d} \bar{\rho}$. Then, the probability measure $\gamma=(X, Y)_{\sharp} \mathrm{d} \bar{\rho}$ satisfies $\mathbb{P}_{x \sharp} \gamma=f$ and $\mathbb{P}_{y_{\sharp}} \gamma=g$; hence

$$
W_{1}(f, g) \leqslant \int_{\mathbb{R}^{d} \times \mathbb{R}^{d}}|x-y| \mathrm{d} \gamma(x, y)=\int_{\mathbb{R}^{d}}|X-Y| \mathrm{d} \bar{\rho}(x) .
$$

Proposition 3.4 (cf. Villani [25, p. 207]). Let $\mu, \nu$ be two probability measures. The Wasserstein distance of order one between $\mu$ and $\nu$ satisfies

$$
W_{1}(\mu, \nu)=\sup _{\operatorname{Lip}(\phi) \leqslant 1} \int_{\mathbb{R}^{d}} \phi(x)(\mathrm{d} \mu(x)-\mathrm{d} \nu(x)) .
$$

\section{The main stability estimate}

In the following we consider probability measures instead of bounded positive measures. This is not a real restriction since we pass from one case to the other just by a rescaling.

Before giving a stability estimate in Proposition 4.2, we prove a technical lemma, in which we derive an estimate on the difference between two vector fields generated by different solutions.

Lemma 4.1. Let $V$ satisfy $(\mathbf{V})$ and $\eta$ satisfy $(\boldsymbol{\eta})$. Let $r, s \in \mathcal{P}\left(\mathbb{R}^{d}\right)^{k}$. For any $i \in\{1, \ldots, k\}$, we have the following estimate

$$
\left\|V^{i}\left(t, x, r * \eta_{t}^{i}\right)-V^{i}\left(t, x, s * \eta_{t}^{i}\right)\right\|_{\mathbf{L}^{\infty}} \leqslant \operatorname{Lip}_{r}\left(V^{i}\right) \operatorname{Lip}_{x}\left(\eta^{i}\right) \mathscr{W}_{1}(r, s) .
$$

In the previous lemma, the quantity $\mathscr{W}_{1}(r, s)$ on the right hand side could be infinite. If we restrict ourselves to bounded positive measures with first moment finite, then the quantity above is always finite.

Proof. The proof follows from Proposition 3.4 on the Wasserstein distance. Note first that in the case $\operatorname{Lip}\left(\eta^{i, j}\right)=0$ then $\eta^{i, j}$ is constant and we have $\left(r^{j}-s^{j}\right) * \eta^{i, j}(x)=0 \leqslant \operatorname{Lip}\left(\eta^{i, j}\right) W_{1}\left(r^{j}, s^{j}\right)$. Now, in the case $\operatorname{Lip}\left(\eta^{i, j}\right) \neq 0$, thanks to Proposition 3.4, we have

$$
\begin{aligned}
\left(r^{j}-s^{j}\right) * \eta^{i, j}(x) & =\int_{\mathbb{R}^{d}} \eta^{i, j}(x-y)\left(\mathrm{d} r^{j}(y)-\mathrm{d} s^{j}(y)\right) \\
& =\operatorname{Lip}\left(\eta^{i, j}\right) \int_{\mathbb{R}^{d}} \frac{\eta^{i, j}(x-y)}{\operatorname{Lip}\left(\eta^{i, j}\right)}\left(\mathrm{d} r^{j}(y)-\mathrm{d} s^{j}(y)\right) \\
& \leqslant \operatorname{Lip}\left(\eta^{i, j}\right) \sup _{\operatorname{Lip}(\phi) \leqslant 1} \int_{\mathbb{R}^{d}} \phi(y)\left(\mathrm{d} r^{j}(y)-\mathrm{d} s^{j}(y)\right) \\
& =\operatorname{Lip}\left(\eta^{i, j}\right) W_{1}\left(r^{j}, s^{j}\right) .
\end{aligned}
$$


As we obtain the same estimate for $-\left(r^{j}-s^{j}\right) * \eta^{i, j}(x)$, we can conclude that

$$
\begin{aligned}
\left\|V^{i}\left(t, x, r * \eta^{i}\right)-V^{i}\left(t, x, s * \eta^{i}\right)\right\|_{\mathbf{L}^{\infty}} & \leqslant \operatorname{Lip}_{r}\left(V^{i}\right)\left\|(r-s) * \eta^{i}\right\|_{\mathbf{L}^{\infty}} \\
& \leqslant \operatorname{Lip}_{r}\left(V^{i}\right) \operatorname{Lip}\left(\eta^{i}\right) \mathscr{W}_{1}(r, s) .
\end{aligned}
$$

Let $r, s \in \mathbf{L}^{\infty}\left([0, T], \mathcal{P}\left(\mathbb{R}^{d}\right)^{k}\right)$. We want to compare the following equations, in which the nonlocal vector fields has been replaced by fixed vector fields. In this way, the system is made of the two decoupled equations:

$$
\begin{array}{lll}
\text { for all } i \in\{1, \ldots, k\} & \partial_{t} \rho^{i}+\operatorname{div}\left(\rho^{i} V^{i}\left(t, x, \eta^{i} * r\right)\right)=0, & \rho^{i}(0, \cdot)=\bar{\rho}^{i}, \\
\text { for all } i \in\{1, \ldots, k\} & \partial_{t} \sigma^{i}+\operatorname{div}\left(\sigma^{i} U^{i}\left(t, x, \nu^{i} * s\right)\right)=0, & \sigma^{i}(0, \cdot)=\bar{\sigma}^{i} .
\end{array}
$$

Proposition 4.2. Assume $V, U$ satisfy (V) and $\eta, \nu$ satisfy $(\boldsymbol{\eta})$. Let $\bar{\rho}, \bar{\sigma}$ be two probability measures such that for any $i,\left\|\bar{\rho}^{i}\right\|_{\mathcal{M}}=\left\|\bar{\sigma}^{i}\right\|_{\mathcal{M}}$. Let $r, s \in$ $\mathbf{L}^{\infty}\left([0, T], \mathcal{P}\left(\mathbb{R}^{d}\right)^{k}\right)$. If $\rho$ and $\sigma$ are Lagrangian solutions of $(4.1)$ associated to the initial conditions $\bar{\rho}$ and $\bar{\sigma}$, then we have the estimate:

$$
\begin{aligned}
\mathscr{W}_{1}\left(\rho_{t}, \sigma_{t}\right) \leqslant & e^{C t} \mathscr{W}_{1}(\bar{\rho}, \bar{\sigma}) \\
& +C t e^{C t}\left[\sup _{t \in[0, T]} \mathscr{W}_{1}\left(r_{t}, s_{t}\right)+\|\eta-\nu\|_{\mathbf{L}^{\infty}}+\|V-U\|_{\mathbf{L}^{\infty}}\right]
\end{aligned}
$$

where $C$ is a constant depending on $\operatorname{Lip}_{x}(V), \operatorname{Lip}_{r}(V), \operatorname{Lip}_{x}(\eta)$ and $\|\bar{\rho}\|_{\mathcal{M}}$.

Furthermore, in the special case $r=\rho$ and $s=\sigma$, we get:

$$
\mathscr{W}_{1}\left(\rho_{t}, \sigma_{t}\right) \leqslant e^{K t} \mathscr{W}_{1}(\bar{\rho}, \bar{\sigma})+K t e^{K t}\left[\|\eta-\nu\|_{\mathbf{L}^{\infty}}+\|V-U\|_{\mathbf{L}^{\infty}}\right],
$$

where $K$ is a constant depending on $\operatorname{Lip}_{x}(V), \operatorname{Lip}_{r}(V), \operatorname{Lip}_{x}(\eta)$ and $\|\bar{\rho}\|_{\mathcal{M}}$.

Note that the estimate above comprises the case $\mathscr{W}_{1}(\bar{\rho}, \bar{\sigma})=\infty$.

Proof. Let $\rho, \sigma$ be two Lagrangian solutions to the Cauchy problem for (1.1) with initial conditions $\bar{\rho}$ and $\bar{\sigma}$ respectively. Let $X, Y$ be the associated ODE flows. For any $t \in[0, T]$, we define the map $X_{t}^{i} \bowtie Y_{t}^{i}: \mathbb{R}^{d} \times \mathbb{R}^{d} \rightarrow \mathbb{R}^{d} \times \mathbb{R}^{d}$ by

$$
X_{t}^{i} \bowtie Y_{t}^{i}(x, y)=\left(X_{t}^{i}(x), Y_{t}^{i}(y)\right), \quad \text { for any }(x, y) \in \mathbb{R}^{d} \times \mathbb{R}^{d} .
$$

Let $\gamma_{0}^{i} \in \Xi\left(\bar{\rho}^{i}, \bar{\sigma}^{i}\right)$ so that $\mathbb{P}_{x \sharp} \gamma_{0}^{i}=\bar{\rho}^{i}$ and $\mathbb{P}_{y_{\sharp}} \gamma_{0}^{i}=\bar{\sigma}^{i}$. Let us define the probability measure $\gamma_{t}^{i}=\left(X_{t}^{i} \bowtie Y_{t}^{i}\right)_{\sharp} \gamma_{0}^{i}$. Then, $\mathbb{P}_{x \sharp} \gamma_{t}^{i}=\rho_{t}^{i}$ and $\mathbb{P}_{y_{\sharp}} \gamma_{t}^{i}=\sigma_{t}^{i}$ so that $\gamma_{t}^{i} \in \Xi\left(\rho_{t}^{i}, \sigma_{t}^{i}\right)$

We fix $R>0$ and we define, for $t \geqslant 0$

$$
\begin{aligned}
Q_{R}(t) & =\sum_{i=1}^{k} \int_{X_{t}^{i}\left(B_{R}\right) \times Y_{t}^{i}\left(B_{R}\right)}|x-y| \mathrm{d} \gamma_{t}^{i}(x, y) \\
& =\sum_{i=1}^{k} \int_{B_{R} \times B_{R}}\left|X_{t}^{i}(x)-Y_{t}^{i}(y)\right| \mathrm{d} \gamma_{0}^{i}(x, y) .
\end{aligned}
$$


Note first that $Q_{R}$ is Lipschitz. Indeed, for $t, s \geqslant 0$ we have

$$
\begin{aligned}
\left|Q_{R}(t)-Q_{R}(s)\right| & \leqslant\left|\sum_{i=1}^{k} \int_{B_{R} \times B_{R}}\left(\left|X_{t}^{i}(x)-Y_{t}^{i}(y)\right|-\left|X_{s}^{i}(x)-Y_{s}^{i}(y)\right|\right) \mathrm{d} \gamma_{0}^{i}(x, y)\right| \\
& \leqslant \sum_{i=1}^{k} \int_{B_{R} \times B_{R}}\left|X_{t}^{i}(x)-Y_{t}^{i}(y)-X_{s}^{i}(x)+Y_{s}^{i}(y)\right| \mathrm{d} \gamma_{0}^{i}(x, y) \\
& \leqslant \sum_{i=1}^{k} \int_{B_{R} \times B_{R}}\left(\left|X_{t}^{i}(x)-X_{s}^{i}(x)\right|+\left|Y_{t}^{i}(y)-Y_{s}^{i}(y)\right|\right) \mathrm{d} \gamma_{0}^{i}(x, y) \\
& \leqslant \sum_{i=1}^{k} \int_{B_{R} \times B_{R}}\left(\left\|V^{i}\right\|_{\mathbf{L}^{\infty}}+\left\|U^{i}\right\|_{\mathbf{L}^{\infty}}\right)|t-s| \mathrm{d} \gamma_{0}^{i}(x, y) \\
& \leqslant\left(\|V\|_{\mathbf{L}^{\infty}}+\|U\|_{\mathbf{L}^{\infty}}\right)\left\|\gamma_{0}\right\|_{\mathcal{M}}|t-s| .
\end{aligned}
$$

Let us assume that $\mathscr{W}_{1}(\bar{\rho}, \bar{\sigma})<\infty$, otherwise the thesis is trivial. Then, by Remark 3.2 , for all $i \in\{1, \ldots, k\}$, we can find a bounded positive measure $\gamma_{0}^{i} \in \Xi\left(\bar{\rho}^{i}, \bar{\sigma}^{i}\right)$ so that

$$
W_{1}\left(\bar{\rho}^{i}, \bar{\sigma}^{i}\right)=\int_{\mathbb{R}^{d} \times \mathbb{R}^{d}}|x-y| \mathrm{d} \gamma_{0}^{i}(x, y) .
$$

Consequently we have, for any $R \geqslant 0, Q_{R}(0) \leqslant \mathscr{W}_{1}(\bar{\rho}, \bar{\sigma})$. Hence, using (4.4), for any $t \geqslant 0$, we have

$$
\begin{aligned}
Q_{R}(t) & \leqslant Q_{R}(0)+\left(\|U\|_{\mathbf{L}^{\infty}}+\|V\|_{\mathbf{L}^{\infty}}\right)\left\|\gamma_{0}\right\|_{\mathcal{M}} t \\
& \leqslant \mathscr{W}_{1}(\bar{\rho}, \bar{\sigma})+\left(\|U\|_{\mathbf{L}^{\infty}}+\|V\|_{\mathbf{L}^{\infty}}\right)\left\|\gamma_{0}\right\|_{\mathcal{M}^{t}} .
\end{aligned}
$$

Thus, for any $t \geqslant 0, Q_{R}(t)$ remains finite when $R \rightarrow \infty$ and since $R \mapsto Q_{R}(t)$ is increasing with respect to $R$, we can define $Q(t)=\lim _{R \rightarrow \infty} Q_{R}(t)$.

Let us now consider $Q$. The same computation as in (4.4) ensures that $Q$ is Lipschitz so we can differentiate for almost every $t$ and obtain

$$
\begin{aligned}
Q^{\prime}(t) \leqslant & \sum_{i=1}^{k} \int_{\mathbb{R}^{d} \times \mathbb{R}^{d}} \mid V^{i}\left(t, X_{t}^{i}(x), r_{t} * \eta_{t}^{i}\left(X_{t}^{i}(x)\right)\right) \\
& -U^{i}\left(t, Y_{t}^{i}(y), s_{t} * \nu_{t}^{i}\left(Y_{t}^{i}(y)\right)\right) \mid \mathrm{d} \gamma_{0}^{i}(x, y) \\
\leqslant & \sum_{i=1}^{k} \int_{\mathbb{R}^{d} \times \mathbb{R}^{d}}\left(\left|V^{i}\left(t, X_{t}^{i}(x), r_{t} * \eta_{t}^{i}\left(X_{t}^{i}(x)\right)\right)-V^{i}\left(t, Y_{t}^{i}(y), r_{t} * \eta_{t}^{i}\left(X_{t}^{i}(x)\right)\right)\right|\right. \\
& +\left|V^{i}\left(t, Y_{t}^{i}(y), r_{t} * \eta_{t}^{i}\left(X_{t}^{i}(x)\right)\right)-V^{i}\left(t, Y_{t}^{i}(y), r_{t} * \eta_{t}^{i}\left(Y_{t}^{i}(y)\right)\right)\right| \\
& +\left|V^{i}\left(t, Y_{t}^{i}(y), r_{t} * \eta_{t}^{i}\left(Y_{t}^{i}(y)\right)\right)-V^{i}\left(t, Y_{t}^{i}(y), s_{t} * \eta_{t}^{i}\left(Y_{t}^{i}(y)\right)\right)\right| \\
& +\left|V^{i}\left(t, Y_{t}^{i}(y), s_{t} * \eta_{t}^{i}\left(Y_{t}^{i}(y)\right)\right)-V^{i}\left(t, Y_{t}^{i}(y), s_{t} * \nu_{t}^{i}\left(Y_{t}^{i}(y)\right)\right)\right| \\
& \left.+\left|V^{i}\left(t, Y_{t}^{i}(y), s_{t} * \nu_{t}^{i}\left(Y_{t}^{i}(y)\right)\right)-U^{i}\left(t, Y_{t}^{i}(y), s_{t} * \nu_{t}^{i}\left(Y_{t}^{i}(y)\right)\right)\right|\right) \mathrm{d} \gamma_{0}^{i}(x, y) .
\end{aligned}
$$

Note that

$$
\begin{aligned}
\left|V^{i}\left(t, y, r_{t} * \eta_{t}^{i}(x)\right)-V^{i}\left(t, y, r_{t} * \eta_{t}^{i}(y)\right)\right| & \leqslant \operatorname{Lip}_{r}\left(V^{i}\right) \operatorname{Lip}\left(r_{t} * \eta_{t}^{i}\right)|x-y| \\
& \leqslant \operatorname{Lip}_{r}\left(V^{i}\right)\left\|r_{t}\right\|_{\mathcal{M}} \operatorname{Lip}\left(\eta^{i}\right)|x-y|
\end{aligned}
$$


Using Lemma 4.1 we obtain

$$
\begin{aligned}
Q^{\prime}(t) \leqslant & \sum_{i=1}^{k} \int_{\mathbb{R}^{d} \times \mathbb{R}^{d}}\left(\operatorname{Lip}_{x}\left(V^{i}\right)+\operatorname{Lip}_{r}\left(V^{i}\right)\left\|r_{t}\right\|_{\mathcal{M}} \operatorname{Lip}\left(\eta^{i}\right)\right)\left|X_{t}^{i}(x)-Y_{t}^{i}(y)\right| \mathrm{d} \gamma_{0}^{i}(x, y) \\
& +\sum_{i=1}^{k} \int_{\mathbb{R}^{d} \times \mathbb{R}^{d}} \operatorname{Lip}_{r}\left(V^{i}\right) \operatorname{Lip}\left(\eta^{i}\right) \mathscr{W}_{1}\left(r_{t}, s_{t}\right) \mathrm{d} \gamma_{0}^{i}(x, y) \\
& +\sum_{i=1}^{k} \int_{\mathbb{R}^{d} \times \mathbb{R}^{d}} \operatorname{Lip}_{r}\left(V^{i}\right)\left|s_{t} *\left(\eta_{t}^{i}-\nu_{t}^{i}\right)\right| \mathrm{d} \gamma_{0}^{i}(x, y) \\
& +\sum_{i=1}^{k} \int_{\mathbb{R}^{d} \times \mathbb{R}^{d}}\left\|V^{i}-U^{i}\right\|_{\mathbf{L}^{\infty}} \mathrm{d} \gamma_{0}^{i}(x, y) \\
\leqslant & C\left[Q(t)+\mathscr{W}_{1}\left(r_{t}, s_{t}\right)+\|\eta-\nu\|_{\mathbf{L}^{\infty}}+\|U-V\|_{\mathbf{L}^{\infty}}\right] .
\end{aligned}
$$

Taking the sup in time of $\mathcal{W}_{1}\left(r_{t}, s_{t}\right)$ on the right-hand side and applying Gronwall Lemma, we get

$$
Q(t) \leqslant e^{C t} Q(0)+C t e^{C t}\left(\sup _{\tau \in[0, t]} \mathscr{W}_{1}\left(r_{\tau}, s_{\tau}\right)+\|\eta-\nu\|_{\mathbf{L}^{\infty}}+\|U-V\|_{\mathbf{L}^{\infty}}\right) .
$$

Note now that, thanks to Remark 3.3, for any $t \geqslant 0$

$$
\mathscr{W}_{1}\left(\rho_{t}, \sigma_{t}\right) \leqslant Q(t)
$$

Furthermore, we have chosen $\gamma_{0}$ in an optimal way thanks to Remark 3.2, and so $Q(0)=\mathscr{W}_{1}(\bar{\rho}, \bar{\sigma})$. Hence we obtain, for any $t \in[0, T]$ :

$$
\begin{aligned}
\mathscr{W}_{1}\left(\rho_{t}, \sigma_{t}\right) \leqslant & e^{C t} \mathscr{W}_{1}(\bar{\rho}, \bar{\sigma}) \\
& +C t e^{C t}\left(\sup _{\tau \in[0, t]} \mathscr{W}_{1}\left(r_{\tau}, s_{\tau}\right)+\|\eta-\nu\|_{\mathbf{L}^{\infty}}+\|U-V\|_{\mathbf{L}^{\infty}}\right),
\end{aligned}
$$

which is the expected result (4.2).

In the particular case $r=\rho$ and $s=\sigma$, applying (4.6) to (4.5) we obtain

$$
Q^{\prime}(t) \leqslant 2 C Q(t)+C\left(\|\eta-\nu\|_{\mathbf{L}^{\infty}}+\|U-V\|_{\mathbf{L}^{\infty}}\right) .
$$

Applying Gronwall Lemma, we finally obtain $Q(t) \leqslant e^{2 C t} Q(0)+C t e^{2 C t}$ $\left(\|\eta-\nu\|_{\mathbf{L}^{\infty}}+\|U-V\|_{\mathbf{L}^{\infty}}\right)$, which is (4.3).

\section{Proof of the main theorem}

The proof of Theorem 1.1 is based on the following idea: let us fix the nonlocal term and, instead of (1.1), we study the Cauchy problem

$$
\partial_{t} \rho+\operatorname{div}(\rho V(t, x, r * \eta))=0, \quad \rho(0)=\bar{\rho},
$$

where $r$ is a given application. We want to use a fixed point argument. We consider here probability measures. In the more general case of positive measures with the same total mass, by rescaling we are back to the case of probability measures. 
Let us introduce the application

$$
\mathscr{Q}:\left\{\begin{aligned}
r & \mapsto \rho \\
\mathscr{X} & \rightarrow \mathscr{X}
\end{aligned}\right\},
$$

where we consider the space $\mathscr{X}=\mathbf{L}^{\infty}\left([0, T], \mathcal{P}\left(\mathbb{R}^{d}\right)^{k}\right)$ for $T$ chosen in such a way that:

(a) The space $\mathscr{X}$ is equipped with a distance $d$ that makes $\mathscr{X}$ complete: for $\mu, \nu \in \mathscr{X}$, we define

$$
d(\mu, \nu)=\sup _{t \in[0, T]} \mathscr{W}_{1}\left(\mu_{t}, \nu_{t}\right)
$$

(b) The application $\mathscr{Q}$ is well-defined: the Lagrangian solution $\rho \in \mathscr{X}$ to (5.1) exists and is unique (for a fixed $r$ ). Indeed, let $X_{t}$ be the ODE flow associated to $V\left(t, x, r_{t} * \eta_{t}\right)$, then we can define $\rho_{t}=X_{t \sharp} \bar{\rho}$. Since $\bar{\rho}$ is a positive measure, then so is $\rho_{t}$.

(c) The application $\mathscr{Q}$ is a contraction: this is given by Proposition 4.2. Indeed, let $r, s$ in $\mathbf{L}^{\infty}\left([0, T], \mathcal{M}^{+}\left(\mathbb{R}^{d}\right)^{k}\right)$ and denote $\rho=\mathscr{Q}(r), \sigma=\mathscr{Q}(s)$ the associated solutions to (5.1). Note that $\rho$ and $\sigma$ have the same initial condition. Thanks to Proposition 4.2, we obtain the contraction estimates

$$
\sup _{[0, T]} \mathscr{W}_{1}\left(\rho_{t}, \sigma_{t}\right) \leqslant C T e^{C T} \sup _{[0, T]} \mathscr{W}_{1}\left(r_{t}, s_{t}\right)
$$

where $C$ depends only on $\operatorname{Lip}_{x}(V), \operatorname{Lip}_{r}(V), \operatorname{Lip}_{x}(\eta)$ and $\|\bar{\rho}\|_{\mathcal{M}}$.

Hence, for $T$ small enough, by the Banach fixed point Theorem we obtain existence and uniqueness in $\mathscr{X}$ of a Lagrangian solution to (1.1) for $t \in[0, T]$. As $\left\|\rho_{T}\right\|_{\mathcal{M}}=\|\bar{\rho}\|_{\mathcal{M}}$ the coefficient $C$ in (5.3) does not depend on time and we can iterate the procedure. Thus we have existence and uniqueness for all positive times.

This concludes the proof of Theorem 1.1. Observe that uniqueness can be also obtained directly by the stability estimate (4.3) in the particular case $V^{i}=U^{i}, \eta^{i}=\nu^{i}, \bar{\rho}=\bar{\sigma}$.

\section{References}

[1] Ambrosio, L., Gangbo, W.: Hamiltonian ODEs in the Wasserstein space of probability measures. Commun. Pure Appl. Math. 61(1), 18-53 (2008)

[2] Armbruster, D., Degond, P., Ringhofer, C.: A model for the dynamics of large queuing networks and supply chains. SIAM J. Appl. Math. 66(3), 896-920 (2006, electronic)

[3] Armbruster, D., Marthaler, D., Ringhofer, C., Kempf, K., Jo, T.: A continuum model for a re-entrant factory. Oper. Res. 54(5), 933-950 (2006)

[4] Bellomo, N., Dogbe, C.: On the modeling of traffic and crowds: a survey of models, speculations, and perspectives. SIAM Rev. 53(3), 409-463 (2011) 
[5] Carrillo, J.A., DiFrancesco, M., Figalli, A., Laurent, T., Slepčev, D.: Global-intime weak measure solutions and finite-time aggregation for nonlocal interaction equations. Duke Math. J. 156(2), 229-271 (2011)

[6] Colombo, R., Lécureux-Mercier, M.: An analytical framework to describe the interactions between individuals and a continuum. J. Nonlinear Sci. 22, 39-61 (2012)

[7] Colombo, R.M., Garavello, M., Lécureux-Mercier, M.: A class of non-local models for pedestrian traffic. Mathematical Models and Methods in Applied Sciences (M3AS) (2012, to appear)

[8] Colombo, R.M., Herty, M., Mercier, M.: Control of the continuity equation with a non local flow. ESAIM Control Optim. Calc. Var. 17(2), 353-379 (2011)

[9] Colombo, R.M., Lécureux-Mercier, M.: Nonlocal crowd dynamics models for several populations. Acta Mathematica Scientia 32(1), 177-196 (2012)

[10] Coron, J., Kawski, M., Wang, Z.: Analysis of a conservation law modeling a highly re-entrant manufacturing system. Discrete Contin. Dyn. Syst. Ser. B 14(4), 1337-1359 (2010)

[11] Coscia, V., Canavesio, C.: First-order macroscopic modelling of human crowd dynamics. Math. Models Methods Appl. Sci. 18(suppl.), 1217-1247 (2008)

[12] Cristiani, E., Piccoli, B., Tosin, A.: Multiscale modeling of granular flows with application to crowd dynamics. Multiscale Model. Simul. 9(1), 155-182 (2011)

[13] Dafermos, C.M.: Hyperbolic conservation laws in continuum physics, Grundlehren der Mathematischen Wissenschaften, vol. 325, 2nd edn. Springer, Berlin (2005).

[14] Di Francesco, M., Markowich, P.A., Pietschmann, J., Wolfram, M.: On the Hughes' model for pedestrian flow: the one-dimensional case. J. Differ. Equ. 250(3), 1334-1362 (2011)

[15] DiPerna, R.J., Lions, P.-L.: Ordinary differential equations, transport theory and Sobolev spaces. Invent. Math. 98(3), 511-547 (1989)

[16] Helbing, D., Johansson, A. and Al-Abideen, H.Z.: Dynamics of crowd disasters: An empirical study. Phys. Rev. E 75, 046109 (2007)

[17] Hughes, R.L.: A continuum theory for the flow of pedestrians. Transp. Res. Part B Methodol. 36(6), 507-535 (2002)

[18] Hughes, R.L.: The flow of human crowds. Annu. Rev. Fluid Mech. 35, 169-182 (2003)

[19] Loeper, G.: Uniqueness of the solution to the Vlasov-Poisson system with bounded density. J. Math. Pures Appl. (9) 86(1), 68-79 (2006)

[20] Maury, B., Roudneff-Chupin, A., Santambrogio, F.: A macroscopic crowd motion model of the gradient-flow type. Math. Models Methods Appl. Sci. 20(10), 1787-1821 (2010) 
[21] Maury, B., Roudneff-Chupin, A., Santambrogio, F., Juliette, V.: Handling congestion in crowd motion modeling. Netw. Heterog. Media 6(3), 485-519 (2011)

[22] Piccoli, B., Rossi, F.: Transport equation with nonlocal velocity in wasserstein spaces: existence, uniqueness and numerical schemes. (2011, preprint)

[23] Piccoli, B., Tosin, A.: Time-evolving measures and macroscopic modeling of pedestrian flow. Arch. Ration. Mech. Anal. 1-32 (2010)

[24] Rubinstein, J.: Evolution equations for stratified dilute suspensions. Phys. Fluids A 2(1), 3-6 (1990)

[25] Villani, C.: Topics in optimal transportation. Graduate Studies in Mathematics, vol. 58. American Mathematical Society, Providence (2003)

[26] Zumbrun, K.: On a nonlocal dispersive equation modeling particle suspensions. Quart. Appl. Math. 57(3), 573-600 (1999)

Gianluca Crippa

Departement Mathematik und Informatik

Universität Basel

Rheinsprung 21

4051 Basel

Switzerland

e-mail: gianluca.crippa@unibas.ch

Magali Lécureux-Mercier

Technion-Israel Institute of Technology

Amado Building

Haifa 32000

Israel

e-mail: mercier@tx.technion.ac.il

Received: 19 December 2011.

Accepted: 13 March 2012. 